\title{
Analysis of Thermal Degradation in the Annealing Process of Colored Parts Built by Extrusion-Based 3D Printing
}

\author{
K. Castro ${ }^{1}$, L. Santana ${ }^{2}$, J. Lino ${ }^{3}$ \\ ${ }^{1}$ University of Debrecen, Faculty of Engineering, leocastroskp18@gmail.com \\ 2University of Porto, Faculty of Engineering, leonardosantana29@gmail.com \\ 3University of Porto, Faculty of Engineering, falves@fe.up.pt
}

\begin{abstract}
Abstrac. With the ever-increasing request of light materials, poly lactic (acid) PLA, have got much in consideration. Low-cost PLA materials have risen its use. Those possess some benefits but nevertheless insufficient mechanical strength. The printed PLA objects have a stumbling block for practical applications. Thus, annealing is an interested alternative to make $3 D$ printed objects strong. This thermal treatment can significantly develop investigational studies and offer technical data. Hence the purpose of this paper is study and discuss how to increase the flexural strength through annealing process. Geometry distortions and color degradation will be analyzed. Differential Scanning Calorimetry, Taguchi Method (TM) and variance (ANOVA) were applied as part of the design experiments and analysis. Twenty-seven printed specimens were tested and factors as temperature, time and color were selected.
\end{abstract}

Keywords: Poli-lactic (Acid), Differential Scanning Calorimetry, Analysis of Variance, Taguchi Method.

\section{Introduction}

Due to the development of open source projects, there is not any standard which regularize technical properties of filaments used to in FDM (Fused deposition modelling) process. Many users set up parameters through experimental tests [1]. Most of the free-code 3D print equipment turn out interesting to the field of investigation, due to flexibility, capacity, and material as PLA-Poly lactic (acid). Some studies were developed to corroborate the properties of PLA filaments [2] [3] [4].

Thermally, PLA shows a glass transition about (55 to 65$){ }^{\circ} \mathrm{C}[5]$ and fusion temperature of $(170-180)^{\circ} \mathrm{C}$ [3]. PLA can transit between an amorphous or semi crystalline polymer. When it is semi crystalline it has a melting temperature between 170 and $180^{\circ} \mathrm{C}$. PLA is widely used in some industries although the lowest impact resistance and tensile and flexural strength [6].

Nevertheless, lack of standardization and information about how to improve mechanical properties without geometrical and color distortion, makes this subject interesting for academic research.

Annealing of materials is used to decrease internal stress in objects and make those objects mechanically stronger, temperature resistant, less brittle, ductile, and less prone to warping [7]. 
DOI: 10.21791/IJEMS.2020.3.13.

An oven annealing temperature was settled at $70^{\circ} \mathrm{C}$, above the glass transition but bellow the melting point. The specimens were treated for 1 hour and then leave it to cool. The results showed an increase of $40 \%$ in mechanical strength; however, it was evident the shrink in the $\mathrm{X}$ and $\mathrm{Y}$ directions, expansion in the $\mathrm{Z}$ direction and warped around the printed surface [7].

Annealing at $75^{\circ} \mathrm{C}$ increased tensile and compressive strength compared to samples tested at room temperature. The annealing effects on crystallinity relief residual stress, and make better inter-layer bonding, which causes changes in mechanical properties of 3D printed specimens [8].

Into some areas of engineering field and biomedical, 3D printed colors are used to get replicas of a real stress. Alteration in color appearance, may be perceived when 3D prints are exposed to annealing process and infiltration agents [9].

Temperature, time, and geometrical tolerance are essential in novel process as freeze nano 3D printing, where the dropped solidification, heat transfer, and time prediction behaviour is crucial [10].

As it can be seen, most of the available literature presents optimization of mechanical properties by considering controllable parameters such: extrusion temperature, print velocity, inner structure, layer height, material, and post processing. However, there is still little information regarding the influence of annealing to geometrical and color properties, thus this being the paper's intention.

\section{Materials and Methods}

Experimentally this work was divided in two groups of analysis Figure 1. The first group make a thermal analysis. The second group study the behaviour and alterations of the 3D printed specimens.

\subsection{Flexion test specimens, Materials, Equipment and Thermal analysis}

The flexion test specimen was prepared according to ASTM D790. PLA 'filament-PM', $1.75 \mathrm{~mm}$ was used. A 3D printer (Ender 3 by Creality3D) with a closed print chamber was operated. Data acquisition equipment 'NOVOS' measured a real approximation of temperature on the bed and the tip extruder. POL-EKD Apartura Incubator STD, used for annealing process. Colorimeter PCE-CSM 5, measured the differences of color before and after annealing treatment. MECMESIN multi test 2.5-dv, tensile and compression tester. Digital micrometre Mitutoyo, for measuring the width and thickness of specimens. 


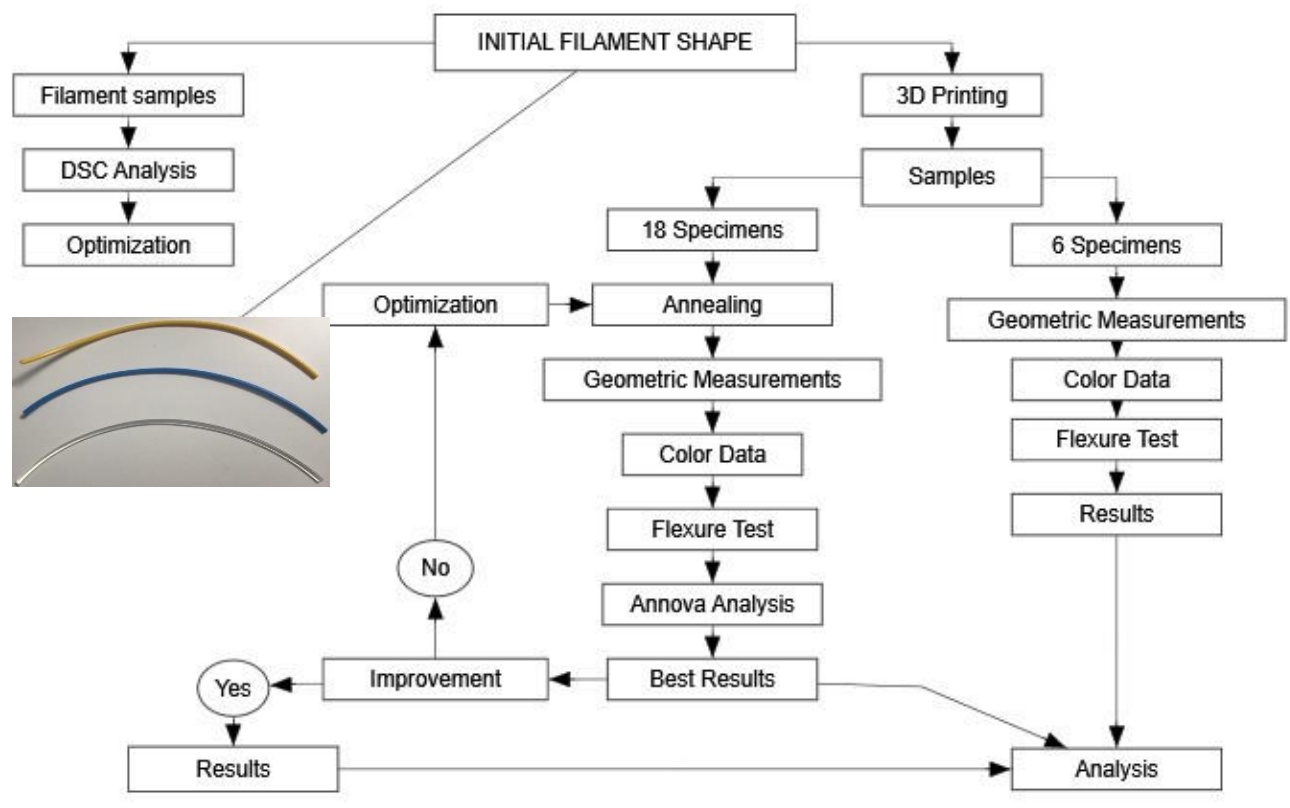

Figure 1 Methodology of Investigation (own source)

The DSG (Differential Scanning Calorimetry) analysis identified the PLA transitions of states as a function of temperature, as Tg-cooling, Tcr- Crystallization and Tf-Fusion. SDT Q6Ta Instruments was operated as equipment. The Figure 2 identify important temperatures for annealing in this study (50, $90,130){ }^{\circ}$ Celsius. A thermal treatment between these temperatures will reorganize the internal crystalline structure, generating bigger grains setting up stronger objects [7].

\subsection{D-Printing Process and Color Analysis}

Printed parameters were setup as Layer height: $0.2 \mathrm{~mm}$, Line width: $0.4 \mathrm{~mm}$, Line wall: 5, Platform temperature: $60^{\circ} \mathrm{C}$, Infill density: $100 \%$, Printing extrude temperature: $215^{\circ} \mathrm{C}$, Material flow rate: $102 \%$, Print speed: $60 \mathrm{~mm} / \mathrm{s}$, Infill speed: $60 \mathrm{~mm} / \mathrm{s}$, Inner/wall speed: $30 \mathrm{~mm} / \mathrm{s}$, Print cooling: Enable, Fan speed: $100 \%$. The specimens were stick off at $30^{\circ} \mathrm{C}$ of bed temperature. Controlling the temperature in the chamber can avoid internal stresses during solidification and cooling.

In this study, spectral values of printed specimens were compared, in terms of CIE L*a*b color shifts and color difference $\mathrm{dE}^{*} \pm 0.500$ tolerance equation 1, with CQCS3 SOFTWARE version 3.4.3.

The collected spectral data was converted into $\mathrm{L}^{*} \mathrm{a}$ b b values of color coordinates in CIELAB color system, where $\mathrm{L}^{*}$ represents the lightness from luminance value while $\mathrm{a}^{*}$ (redness-greenness plane) and $\mathrm{b}^{*}$ (yellowness-blueness plane) symbolize the chromatic value of the color.

Difference color equation: $d E *=\sqrt{d L^{* 2}+d a^{* 2}+d b^{* 2}}$

\subsection{Taguchi Method}

Considering that customer's perception of quality is the most important factor, many companies look for quality through product and process optimization. This structure study was elaborated as shown Figure 3. The first stage defines the factors and the adequate orthogonal array (OA). The second stage is 
essentially execution of experiments. Third stage deals with data examination, which was done using specialized software, and finally the fourth stage agrees to the corroboration of the experiment [11].

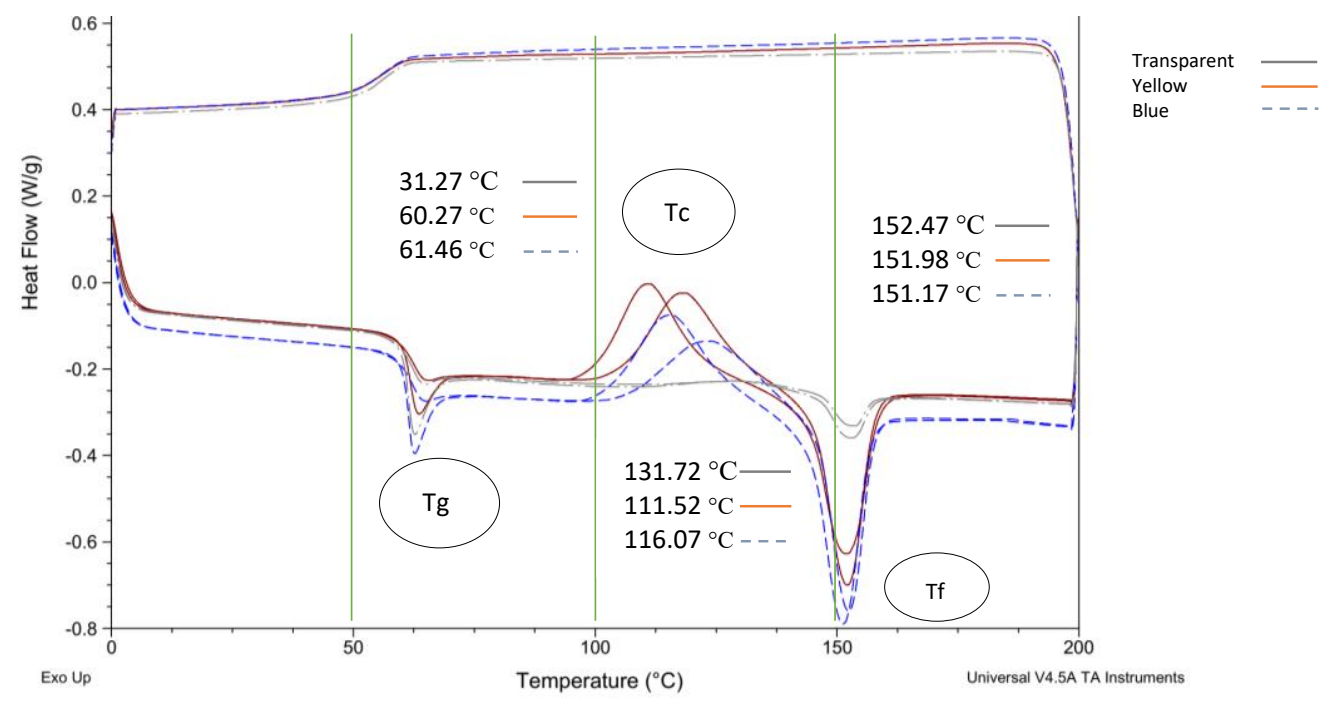

Figure 2 Thermograms obtained by DSC analysis. Tg-cooling, Tcr-crystallization and Tf-fusion temperatures (own source)

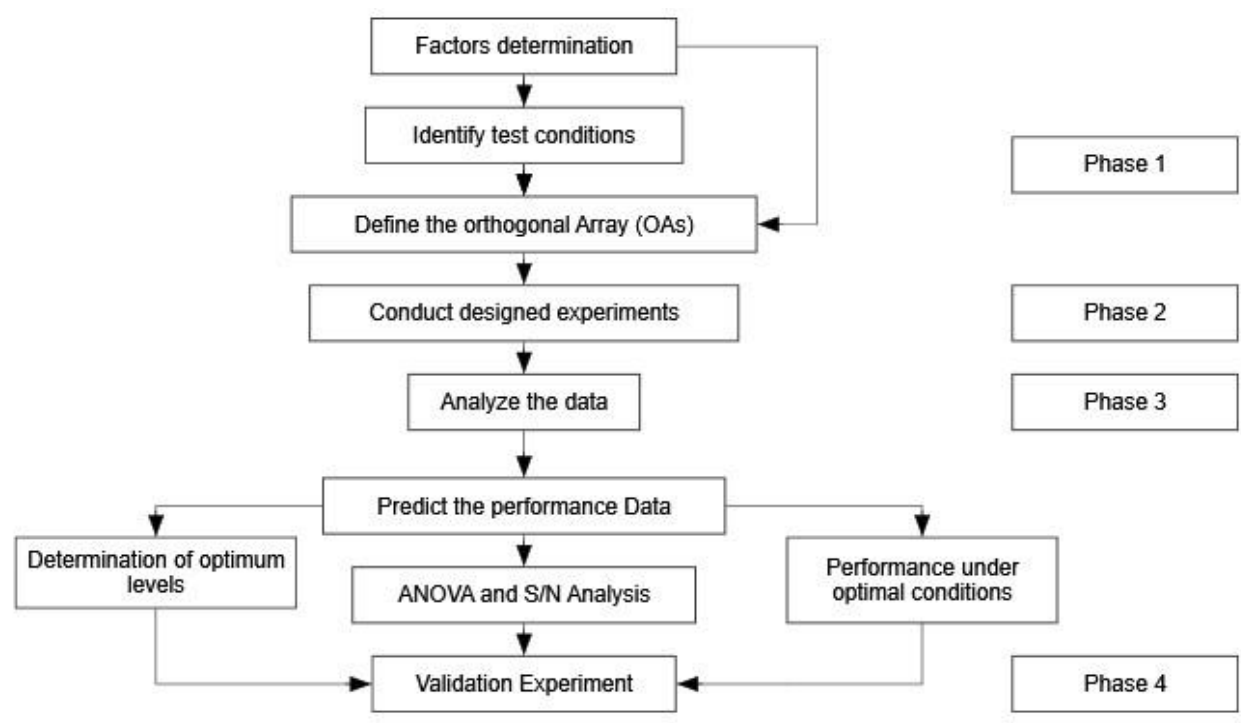

Figure 3 Scheme of the Taguchi Method approach (own source)

Three significant factors were identified: Incubator's Temperature (A), Time (B), and Color (C). Three levels for each factor were recognized as show in Table 1. The knowledge of the process is essential when sectioning the factors and levels to be considered with the experimentations [12].

FACTORS AND LEVELS

\begin{tabular}{lcccc}
\hline \multirow{2}{*}{ FACTORS } & \multirow{2}{*}{ UNITS } & $\mathbf{1}$ & $\mathbf{2}$ & $\mathbf{3}$ \\
\hline A Incubator's temperature & Celsius & 50 & 90 & 130 \\
B Time & Minutes & 20 & 40 & 60 \\
C Color & Type & Transparent & Blue & Yellow \\
\hline
\end{tabular}

Table 1 Taguchi factors and levels 
DOI: 10.21791/IJEMS.2020.3.13.

Defining the appropriate Orthogonal Array (OA) is an essence to assure truthfulness of the statistical model [12]. Thus, a L9 (OA) is chosen for this research. This experimental design shows the array selector for parameters and their interactions. Besides, the measurements and means trials Table 2 .

\begin{tabular}{|c|c|c|c|c|c|c|c|c|c|c|c|c|c|}
\hline $\begin{array}{c}\text { TRIAL } \\
\mathbf{N}^{\circ}\end{array}$ & (A) & (B) & (C) & $\begin{array}{c}\text { S1 } \\
\text { (MPa) }\end{array}$ & $\begin{array}{c}\text { S2 } \\
\text { (MPa) }\end{array}$ & $\begin{array}{c}\text { S mean } \\
(\mathrm{MPa})\end{array}$ & $\begin{array}{c}\mathrm{T} 1 \\
(\mathrm{~mm})\end{array}$ & $\begin{array}{c}\mathrm{T} 2 \\
(\mathrm{~mm})\end{array}$ & $\begin{array}{c}T \text { mean } \\
(\mathrm{mm})\end{array}$ & $\begin{array}{c}\text { W1 } \\
(\mathrm{mm})\end{array}$ & $\begin{array}{c}\text { W2 } \\
(\mathrm{mm})\end{array}$ & $\begin{array}{c}\text { W mean } \\
(\mathrm{mm})\end{array}$ & $\mathrm{dE}^{*}$ \\
\hline 1 & 50 & 20 & Transparent & 91.963 & 96.752 & 94.357 & 3.372 & 3.045 & 3.209 & 12.552 & 12.666 & 12.609 & 1.744 \\
\hline 2 & 50 & 40 & Blue & 106.876 & 105.499 & 106.188 & 3.089 & 3.05 & 3.070 & 12.723 & 12.877 & 12.800 & 2.216 \\
\hline 3 & 50 & 60 & Yellow & 109.546 & 108.359 & 108.952 & 3.043 & 3.068 & $\underline{3.056}$ & 12.902 & 12.611 & 12.757 & $\underline{0.470}$ \\
\hline 4 & 90 & 20 & Blue & 111.136 & 112.791 & 111.964 & 3.427 & 3.418 & 3.423 & 12.902 & 12.611 & 12.757 & 1.484 \\
\hline 5 & 90 & 40 & Yellow & 110.350 & 117.039 & 113.695 & 3.437 & 3.301 & 3.369 & 12.485 & 12.732 & 12.609 & 1.508 \\
\hline 6 & 90 & 60 & Transparent & 100.390 & 119.852 & 110.121 & 3.661 & 3.381 & 3.521 & 12.235 & 12.235 & 12.235 & 2.462 \\
\hline 7 & 130 & 20 & Yellow & 96.050 & 98.198 & 97.124 & 3.567 & 3.586 & 3.577 & 12.274 & 12.235 & 12.255 & 3.468 \\
\hline 8 & 130 & 40 & Transparent & 102.299 & 100.083 & 101.191 & 3.711 & 3.7103 & 3.711 & 12.271 & 12.248 & 12.260 & 7.394 \\
\hline 9 & 130 & 60 & Blue & 106.801 & 99.715 & 103.258 & 3.583 & 3.753 & 3.668 & 12.335 & 12.202 & 12.269 & 4.202 \\
\hline
\end{tabular}

Table 2 Experimental results after thermal exposure: flexural Stress (S), thickness (T) and width (W) mean, shift color difference ( $d E^{*}$ ) based on $L^{*} a^{*} b^{*}$ measurements (own source).

\subsection{Annova Analysis and Performance Data}

F-value is compared to the standard value $\mathrm{F}(0.05,2,2)$, which means at the $5 \%$ of significance level, degrees of freedom (DF) of the factor, and the DF of the error correspondingly. In this study a factor is significant only if the calculated $F$-value is greater than $F(0.05,2,2)=19$. For flexural analysis case, factor (A) is not statistically significant, despite $58 \%$ of influence Table 3.

\begin{tabular}{lccccccc}
\hline Source & DF & S & V & F & $\%$ P & P value & $\mathrm{F}(0.05,2,2)=19$ \\
\hline Time & 2 & 0.1847 & 0.0923 & 3.42 & 21.353 & 0.226 & X \\
Color & 2 & 0.1213 & 0.0600 & 2.25 & 14.023 & 0.308 & $X$ \\
Error & 2 & 0.0540 & 0.0270 & & 6.243 & & \\
& & & & & 100.03 & & \\
Total & 8 & 0.8650 & & & 5 &
\end{tabular}

Table 3 Analysis of Variance flexural stress (own source)

In order to get the prediction model, an analysis of the factors was performed as shown in Figure 4 . For the case of thickness analysis, factor (A) is statistically significant, as $92 \%$ influential factor towards a response. For the case of width, the factor (A) is statistically significant, about $78 \%$ of effect. In Color case, factor (A) is responsible about the $69 \%$ of final response. Factors (B) and (C) do not represent a significance to the result in all the cases.

Agreeing to the Taguchi model, the greatest performance for the experiment can be achieved with the next expression equation 2, [12]:

Optimal result equation: $R o p t=A+B+C-2 *$ Rmean 
The values (A, B, C) for each factor are taken from response means tables. Table 4 shows means and an optimized result of 115.49 , for flexural stress case. On the other hand, $3.034 \mathrm{~mm}$ and $12.896 \mathrm{~mm}$ are the optimized results for thickness and width cases respectively Table 4. A confidence interval (CI) must be calculated for validation procedure as show equation 3.

$$
\text { Confidential interval equation: } C I=\sqrt{F(1, D F E) * V e *\left[\frac{1}{N e f f}+\frac{1}{R}\right]} ; \quad \text { Neff }=\frac{N}{1+V t}
$$

Where F $(1, \mathrm{DFE})$ is taken form tables at $95 \%$ confidence. DFE is the number of DF related to the error, it is 2 . $F(1,2)=18.513$. Ve is the variance of the error for each case of study. For flexural stress case the information can be found in Table 3. $\mathrm{R}=2$, is the number of the test repetitions. Neff is the effective number of replications. $\mathrm{N}=9$, is the total number of trials in this study. $\mathrm{Vt}=2$ it is the total DF of influence factors. Thus, CI: flexural Stress $= \pm 0.645 \mathrm{MPa}$, thickness $= \pm 0.026 \mathrm{~mm}$ and width $= \pm 0.032 \mathrm{~mm}$.

\begin{tabular}{|l|l|l|l|}
\hline Level & Inc Temp(A) & Time(B) & Color(C) \\
\hline 1 & 103.2 & 101.1 & 107.1 \\
\hline 2 & $\underline{111.9}$ & 107.0 & 101.9 \\
\hline 3 & 100.5 & $\underline{107.4}$ & 106.6 \\
\hline Delta & 11.4 & 6.3 & 5.2 \\
\hline Rank & 1 & 2 & 3 \\
\hline
\end{tabular}

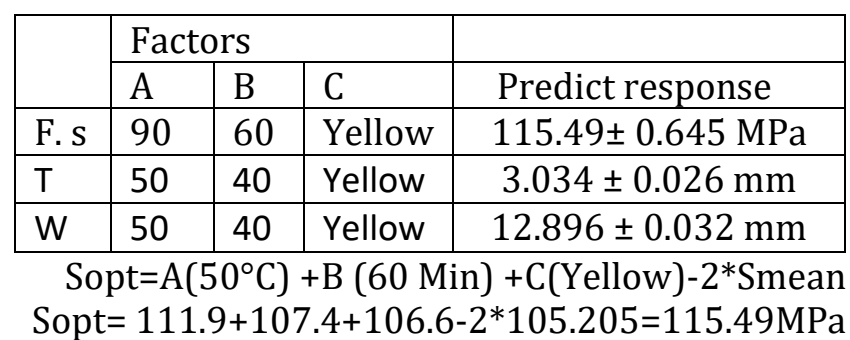

Table 4 Response means for Flexural Stress and predict responses for each case F.s (Flexural stress), T(Thickness), W (width) (own source) 


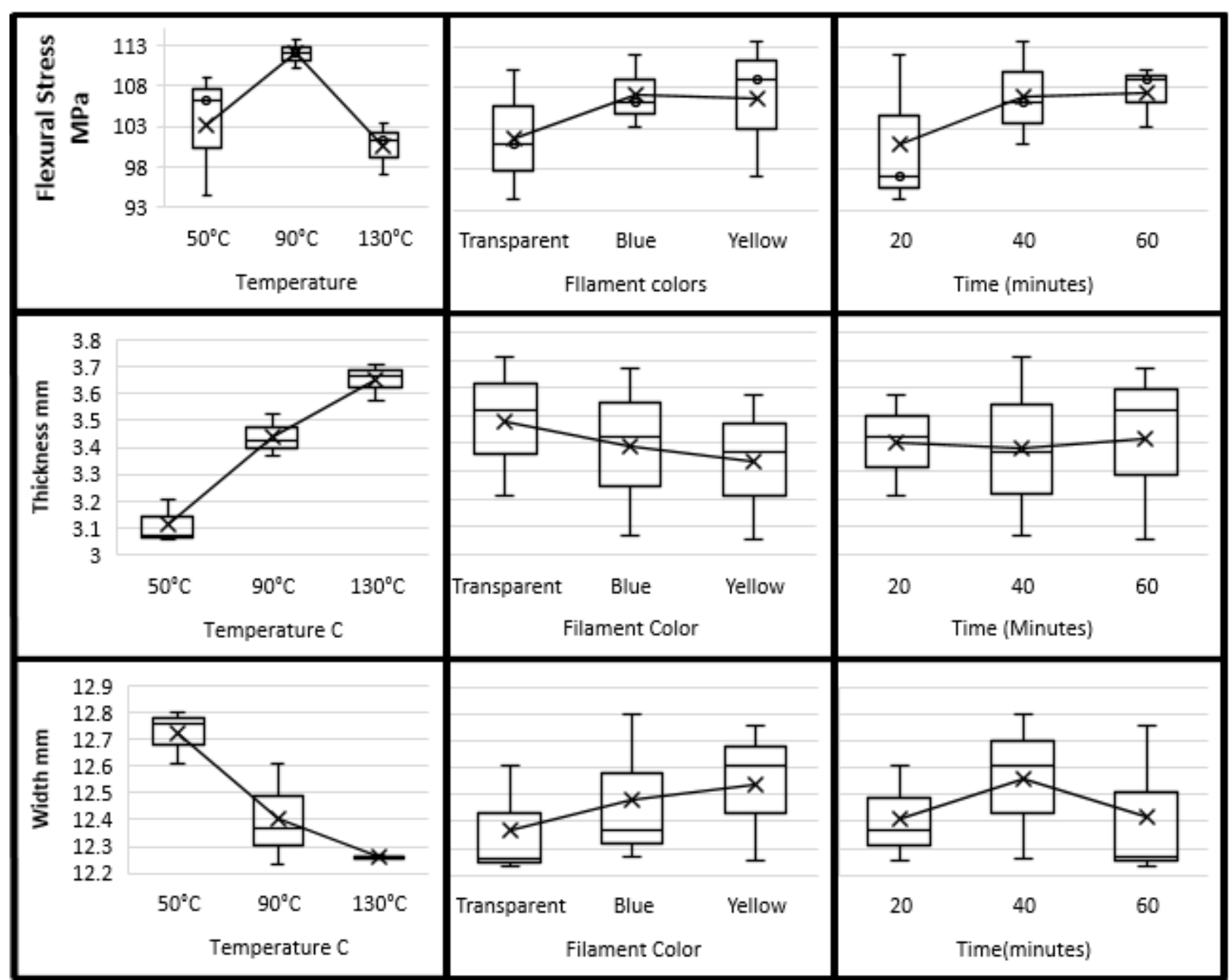

Figure 4 Response of main effect factors for flexural stress, thickness, and width cases
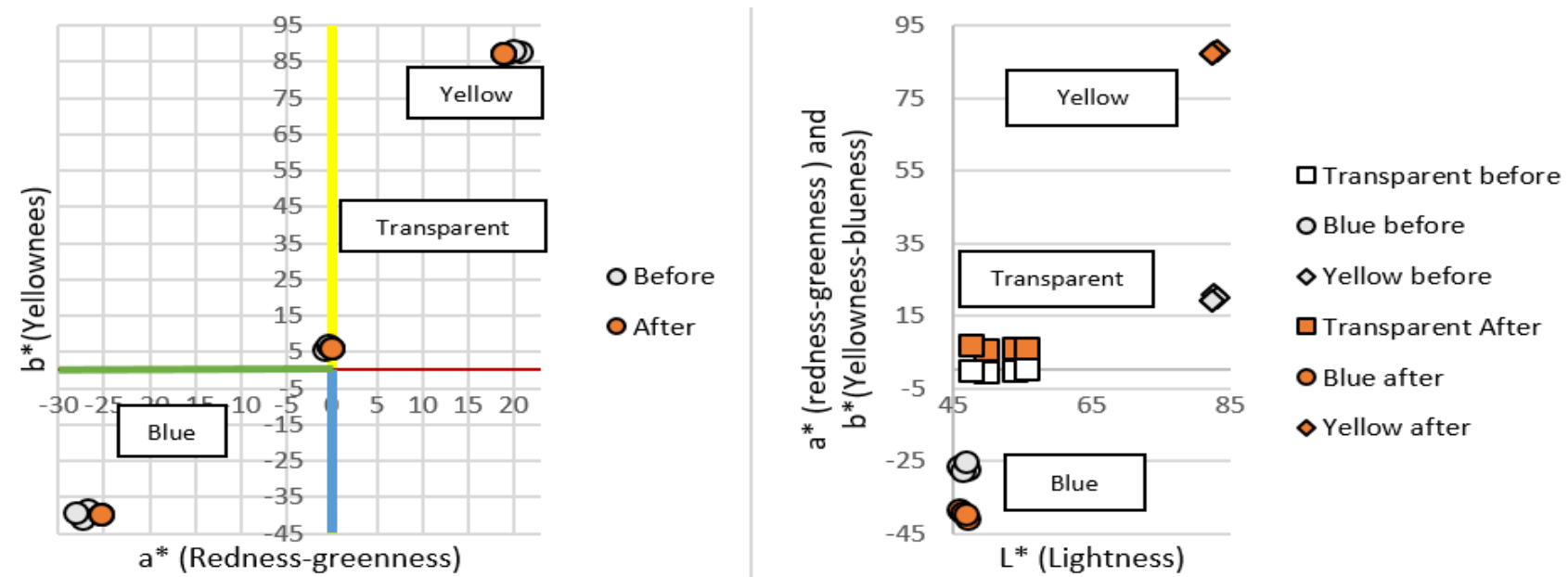

Figure 5 Right. - $a^{*} v s b^{*}$ color printed specimens' measurements. Left. - $L^{*} v s a^{*}$ and $L^{*} v s$ b samples Colored specimens (own source)

The Figure 5 shows the CIELAB $\mathrm{a}^{*} \mathrm{~b}^{*}$ plot, of all the colored printed specimens (Left graph). When comparing the position of the marks before and after annealing. A depreciable shift in chroma can be 
DOI: 10.21791/IJEMS.2020.3.13.

visible. The shift in lightness values L* (Right graph), an appreciable difference can be seen in yellow printed specimens.

\section{Discussion and Conclusions}

Validation experiments were performed for optimal combinations as it can be seen on Table 5. Yet as a temperature below $90^{\circ} \mathrm{C}$ and upper $50^{\circ} \mathrm{C}$, can be used as effect of significant positive results for future investigations. Factor time (B) correlates positively with the result. An increment of flexural stress can be seen in yellow filament test about $11.75 \%$ Figure 6, and insignificant variation in thickness, width and color properties. The values were compared with specimens without annealing treatment.

A judgment can be carried out whit this analysis to verify whether color difference can meet the requirements, after the thermal/time exposure, according to the allowed customer's tolerance and color standard. Thus, this study provides considerations and information to compare, about the behaviour of geometric, color and flexural properties of 3D printed specimens after annealing process.

The characterization of PLA enabled to corroborate thermal properties and relevant transitions of states as a function of temperature. The result shows aspects of semi crystalline polymer but amorphous and crystalline pre-eminence. The filament tested did not lose mass during the experiment.

Correlating the obtained results with previous studies [13], they presented as a result of 1 hour of annealing at (80 and 100) ${ }^{\circ}$ Celsius a flexural strength about (118 and 125) MPa respectively for 3D printed PLA samples. These values are positives but, they do not consider the geometric and color deformation caused by the annealing temperature upper $50^{\circ}$ Celcius.

The results display to 3D printing users, the importance of time and annealing temperature for an increment of the flexural strength without geometric and color significant alterations. The results are base for future investigations, where changes of parameters, equipment, software, materials, filling printing and cost can be implemented, improved, and compared with other technologies.

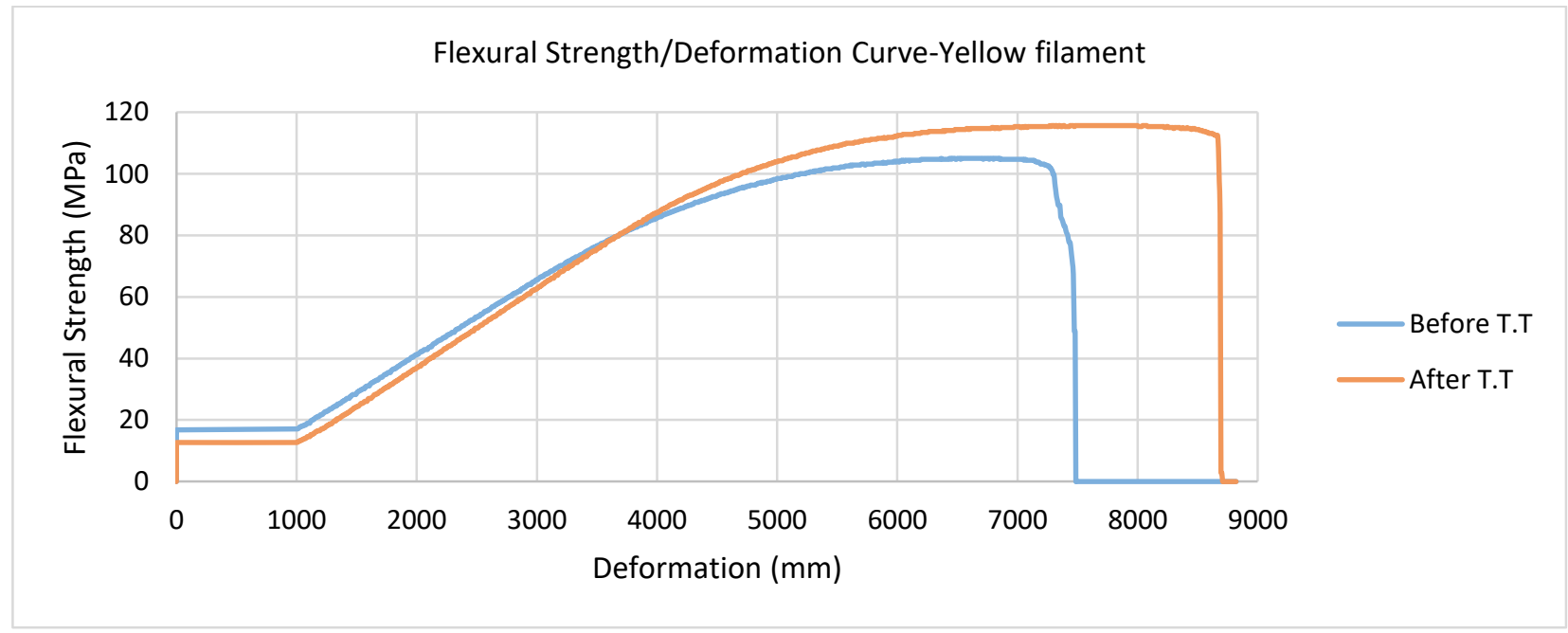

Figure 6 Force/Deformation curve, Before and after annealing, yellow color filament (own source) 


\begin{tabular}{clllllll}
$\begin{array}{c}\text { TRIAL } \\
\mathbf{N}^{\circ}\end{array}$ & $\begin{array}{l}\text { Inc- } \\
\text { Temp }\end{array}{ }^{\circ} \mathrm{C}$ & $\begin{array}{l}\text { Time } \\
\text { (Minutes) }\end{array}$ & Color & $\begin{array}{l}\text { S mean } \\
(\mathrm{MPa})\end{array}$ & $\begin{array}{l}\text { Thickness } \\
(\mathrm{mm})\end{array}$ & $\begin{array}{l}\text { Width } \\
(\mathrm{mm})\end{array}$ & $\mathrm{dE}^{*}$ \\
\hline 12 & 50 & 60 & Transparent & 104.232 & 3.098 & 12.587 & 5.285 \\
11 & 50 & 60 & Blue & 116.723 & 3.061 & 12.481 & 4.274 \\
10 & 50 & 60 & Yellow & $\underline{115.703}$ & $\underline{3.072}$ & $\underline{12.501}$ & $\underline{0.455}$
\end{tabular}

Table 5 Validation experiment Flexural Stress, Thickness, width, and Color Difference (own source)

ACKNOWLEDGEMENTS This study was developed in a collaboration between the University of Porto and University of Debrecen as part of an institutional research internship agreement. The authors would also like to have a special mention to SENESCYT-ECUADOR (Secretaría de Educación Superior, Ciencia,Tecnología e Innovación) and Faculty of mechanical Engineering of University of Porto.

\section{References}

[1] K. Szykiedans and W. Credo, "Mechanical properties of FDM and SLA low cost-cost 3D prints," Procedia Engineering, vol. 136, pp. 257-262, 2016.

[2] C. Page, S. Kreuzer and F. Ansari, "Optimizing 3D printed components: a methodological approach to assessing print parameters on tensile properties," in Proceedings of ANTEC, 2017, pp. 82-88.

[3] L. Santana, J. Lino Alves, A. Da Costa, S. Netto and C. Merlini, "A comparative study between PETG and PLA for 3D Printing through thermal, chemical and mechanical characterization," in Revista Materia, Rio de Janeiro, 2018.

[4] A. Sabino Netto, L. Santana and I. Pereira, "Avaliação de corpos de prova produzidos em PLA por manufatura aditiva por extrusão e moldagem por injeção," in 13 Congreso Iberoamericano de Engenharia Mecanica, Lisboa, 2017.

[5] B. Meng, J. Deng, L. Qing, W. Zhiihua and Y. Wei, "Transparent and ductile poly(latic acid)/ poly (butyl acrylate) (PBA) blend: structure and properties," European Polymer Journal, vol. 48, no. 1, pp. 127-135, January 2012.

[6] B. Wittbrodt and J. M. Pearce, "The effects of PLA Color on Materials of 3-D Printed Components," Additive Manufacturing, vol. 8, pp. 110-116, Octuber 2015.

[7] M. Sarcevic, "ALL3DP," ALL3DP, 20 July 2019. [Online]. Available: https://all3dp.com/2/annealing-pla-prints-for-strength-easy-ways/. [Accessed 1810 2019].

[8] V. Slavković, N. Grujović, A. Dišić and A. Radovanović, "Influence of anenealing and Printing Directions on Mechanical Properties of PLA Shape Memory Polymer Produced by Fused Deposition Modeling.," in Serbian Society of Mechanics, Serbia, 2017.

[9] M. Stanic, B. Lozo and D. Gregor-Svetec, "Colorimetric Properties and Stability of 3D Prints," Rapid Prototyping Journal, vol. 18, no. 2, pp. 120-128, 2 March 2012.

[10] L. J. Segura, G. Zhao, H. Sun and C. Zhou, "Gaussian Process Tensor Responses Emulation for Droplet Solidification in Freeze Nano 3D Printing of Energy Products," in ASME 2019 14th International Manufacturing Science and Engineering Conference., Pennsylvania, 2019. 
[11] K. Castro, L. J. Segura, S. D. Castellanos and J. Lino, "Optimization of geometric quality in a 5 axis machining of curved surfaces in a EN-AW-7075 alloy by taguchi method," in Materials Design and Applications, vol. 65, L. F. d. Silva, Ed., Porto, SRINGER, 2016, pp. 347-360.

[12] K. Ranjit, A Primer on the Taguchi Method, Society of Manufacturing Engineers, 2010.

[13] C. Benwood, A. Anstey, J. Andrzejewski and M. Misra, "Improving the impact streght and heat resistance of 3D printed Models: Structure, property and Processing Correlationships during Fused Deposition Modeling (FDM) of Poly (Lactic Acid)," ACS Omega, vol. 3, no. 4, pp. 4400-4411, 2018. 